\title{
PROPAGATION OF HYPO-ANALYTICITY ALONG BICHARACTERISTICS
}

\begin{abstract}
S. BERHANU
It is shown here the hypo-analytic singularities for solutions propagate along the bicharacteristics of hypo-analytic differential operators of principal type. This generalizes the well-known similar result for analytic differential operators.
\end{abstract}

0. Introduction. In [4] Hormander proved a result concerning the propagation of $C^{\infty}$ singularities of solutions of $P u=f$ for a smooth linear partial differential operator $P$ whose leading symbol is real. The analytic version of this question was treated by Hanges in [3]. In this paper we prove a similar theorem for what we call hypo-analytic differential operators. The paper is organized as follows. In $\S 1$ we discuss the structures we work in and introduce our operators. In $\S 2$ we recall the definition of microlocal hypo-analyticity and give a statement of the main result. $\S 3$ discusses the Fourier transform criterion of microlocal hypo-analyticity due to Baouendi, Chang and Treves [1]. A theorem concerning this criterion is proved in the same section and then used in the proof of our main result.

1. Hypo-analytic differential operators. Our results deal with structures which are a special case of the hypo-analytic structures introduced in [1]. Let $\Omega$ be a $C^{\infty}$ manifold of dimension $m$. A hypoanalytic structure of maximal dimension on $\Omega$ is the data of an open covering $\left(U_{\alpha}\right)$ of $\Omega$ and for each index $\alpha$, of $m C^{\infty}$ functions $Z_{\alpha}^{1}, \ldots, Z_{\alpha}^{m}$ satisfying the following two conditions:

(i) $d Z_{\alpha}^{1}, \ldots, d Z_{\alpha}^{m}$ are linearly independent at each point of $U_{\alpha}$;

(ii) if $U_{\alpha} \cap U_{\beta} \neq \varnothing$, there are open neighbors $O_{\alpha}$ of $Z_{\alpha}\left(U_{\alpha} \cap U_{\beta}\right)$ and $O_{\beta}$ of $Z_{\beta}\left(U_{\alpha} \cap U_{\beta}\right)$ and a holomorphic map $F_{\beta}^{\alpha}$ of $O_{\alpha}$ onto $O_{\beta}$, such that

$$
Z_{\beta}=F_{\beta}^{\alpha} \circ Z_{\alpha} \quad \text { on } U_{\alpha} \cap U_{\beta} \text {. }
$$

We will use the notation $Z_{\alpha}=\left(Z_{\alpha}^{1}, \ldots, Z_{\alpha}^{m}\right): U_{\alpha} \rightarrow C^{m}$. A distribution $h$ defined on an open neighborhood of a point $p_{0}$ of $\Omega$ is called hypo-analytic at $p_{0}$ if there is a local chart $\left(U_{\alpha}, Z_{\alpha}\right)$ of the above type whose domain contains $p_{0}$ and a holomorphic function $\tilde{h}$ defined on 
an open neighborhood of $Z_{\alpha}\left(p_{0}\right)$ in $C^{m}$ such that $h=\tilde{h} \circ Z_{\alpha}$ in a neighborhood of $p_{0}$. By a hypo-analytic local chart we mean an $m+1$-tuple $\left(U, Z^{1}, \ldots, Z^{m}\right)$ [abbreviated $(U, Z)$ ] consisting of an open subset $U$ of $\Omega$ and of $m$ hypo-analytic functions $Z^{1}, \ldots, Z^{m}$ whose differentials are linearly independent at every point of $U$. We note that when the $Z^{j}$ are real valued these structures specialize to the real analytic ones. We introduce the following differential operators on $\Omega$ which are naturally associated with its hypo-analytic structure.

Definition 1.1. A linear differential operator $P$ on $\Omega$ is called a hypo-analytic differential operator if for every open set $U$ and every hypo-analytic function $f$ on $U, P f$ is hypo-analytic on $U$.

The following example shows that Definition 1.1 extends the standard definition in the analytic category.

EXAMPLE. Let $\Omega^{\prime}$ be a real-analytic manifold. The real-analytic structure of $\Omega^{\prime}$ can be viewed as a hypo-analytic structure. A differential operator $P: C^{\infty}\left(\Omega^{\prime}\right) \rightarrow C^{\infty}\left(\Omega^{\prime}\right)$ is said to be analytic if, in terms of local analytic coordinates on $\Omega^{\prime}, P$ is given by

$$
P=\sum_{|\alpha| \leq m_{0}} a_{\alpha}(x) D^{\alpha}
$$

where the $a_{\alpha}$ are real-analytic and $m_{0}$ is some integer. This is the case if and only if $P$ is a hypo-analytic differential operator in the sense of Definition 1.1.

Suppose now $\Omega$ is as before and $P$ is a hypo-analytic differential operator on $\Omega$. Let $(U, Z)$ be a hypo-analytic local chart and assume $U$ is a coordinate neighborhood. Choose vector fields $M_{1}, \ldots, M_{m}$ satisfying $M_{l} Z^{k}=\delta_{l}^{k}$. Since $\left\{M_{1}, \ldots, M_{m}\right\}$ is a linearly independent set, there are smooth functions $a_{\alpha}$ defined on $U$ such that on $U, P=$ $\sum_{|\alpha| \leq m_{0}} a_{\alpha} M^{\alpha}$ for some integer $m_{0}$. For $|\alpha| \leq m_{0}, P Z^{\alpha}=a_{\alpha}$ is hypoanalytic. Conversely, it is easy to see that if in every hypo-analytic local chart $(U, Z) P$ can be written in the above form then $P$ is a hypo-analytic differential operator.

2. Statement of the main result. We continue to work in a hypoanalytic local chart $(U, Z)$ of the maximal structure $\Omega$. Before proceeding to the statement of our theorem, we need to recall Sato's microlocalization as adopted in [1]. In the sequel $\Gamma$ is a nonempty, acute and open cone in $R_{m} \backslash\{0\}$. For $A$ an open subset of $U$, we shall use the notation:

$$
N_{\delta}(A, \Gamma)=\left\{Z(x)+\sqrt{-1} Z_{x}(x) v: x \in A, v \in \Gamma,|v|<\delta\right\} .
$$


Definition 2.1. We denote by $B_{\delta}(A, \Gamma)$ the space of holomorphic functions $f$ in $N_{\delta}(A, \Gamma)$ satisfying:

To every compact subset $K$ of $N_{\delta}(A, \Gamma)$ there are an integer $k \geq 0$ and a constant $c>0$ such that $|f(z)| \leq c(\operatorname{dist}[z, Z(A)])^{-k}$ for all $z$ in $K$.

In [1] it is shown that if $A$ is small enough and $f \in B_{\delta}(A, \Gamma)$ then for every $\psi \in C_{c}^{\infty}(A)$,

$$
\lim _{t \rightarrow+0} \int_{A} f\left(Z(x)+\sqrt{-1} Z_{x}(x) t v\right) \psi(x) d Z(x)
$$

exists and is independent of $v \in \Gamma$. We will denote the distribution we get in the limit by $b f$.

Definition 2.2. Let $u \in D^{\prime}(U)$ and $(x, \xi)$ be a point in $U \times$ $\left(R_{m} \backslash\{0\}\right)$. We say that $u$ is hypo-analytic at $(x, \xi)$ if there are an open neighborhood $A \subseteq U$ of $x, \delta>0$ and a finite collection of nonempty acute open cones $\Gamma_{k}$ in $R_{m} \backslash\{0\}(k=1, \ldots, r)$ such that the following hold: for every $k$ and every $v \in \Gamma_{k}, \xi \cdot v<0$; for each $k$ there is $f_{k} \in B_{\delta}\left(A, \Gamma_{k}\right)$ such that in $A$

$$
u=b f_{1}+\cdots+b f_{k} \text {. }
$$

We remark that the above definition of microlocal hypo-analyticity does not depend on the choice of the chart $(U, Z)$ (see [1]).

Definition 2.3. Let $u \in D^{\prime}(\Omega)$. The hypo-analytic wavefront set of the distribution $u$ is denoted by $W F_{\mathrm{ha}} u$ and is defined as

$$
W F_{\mathrm{ha}} u=\left\{(x, \xi) \in T^{*} \Omega: u \text { is not hypo-analytic at }(x, \xi)\right\}
$$

We now describe the curves in $T^{*} \Omega$ along which hypo-analytic singularities propagate. Let $P_{0}$ be a hypo-analytic differential operator defined near a point $p_{0}$ of $\Omega$.

The previous section tells us that in a hypo-analytic local chart $(U, Z) P$ takes the form $P=\sum_{|\alpha| \leq k} a_{\alpha}(x) M^{\alpha}$ where each $a_{\alpha}(x)$ is a hypo-analytic function on $U$.

After shrinking $U$ if necessary, we may assume that $U$ is the domain of local coordinates $x_{1}, \ldots, x_{m}$. Let $t \rightarrow(x(t), \xi(t))=\gamma(t)$ be a curve in $T^{*} U \backslash 0$ and set

$$
\tilde{\gamma}(t)=(\tilde{x}(t), \tilde{\xi}(t))=\left(Z(x(t)), Z_{x}^{*}(x(t)) \xi(t)\right)
$$

where $Z_{x}^{*}$ denotes the transpose of the inverse of the Jacobian matrix $Z_{x}$. We will use the notation $p(z, \xi)=\sum_{|\alpha|=k} \tilde{a}_{\alpha}(z) \xi^{\alpha}$ where each $a_{\alpha}(x)=\tilde{a}_{\alpha}(Z(x))$ for holomorphic $\tilde{a}_{\alpha}$. 
Definition 2.4. The curve $\gamma(t)$ is said to be a bicharacteristic for $P$ if the equations

$$
\begin{aligned}
& \frac{d \tilde{x}}{d t}=\frac{\partial p}{\partial \zeta}(\tilde{x}(t), \tilde{\xi}(t)) \\
& \frac{d \tilde{\xi}}{d t}=-\frac{\partial p}{\partial z}(\tilde{x}(t), \tilde{\xi}(t))
\end{aligned}
$$

hold.

We can now state the main theorem of this paper.

THEOREM 2.1. Assume $p\left(0, \xi^{0}\right)=0$ and $P$ is of principal type at $\left(0, \xi^{0}\right)$. Suppose $\gamma=\{(x(t), \xi(t))\}$ is a bicharacteristic for $P$ through $(x(0), \xi(0))=\left(0, \xi^{0}\right)$ and that $P u$ is hypo-analytic on $\gamma$. Then either $u$ is hypo-analytic at every point of $\gamma$ or $u$ is not hypo-analytic at any point of $\gamma$.

3. On the Fourier transform criterion of hypo-analyticity. We will work in a hypo-analytic local chart $(U, Z)$ in $\Omega$. We shall assume that the open set $U$ has been contracted so that the map $Z=\left(Z^{1}, \ldots, Z^{m}\right)$ : $U \rightarrow C^{m}$ is a diffeomorphism of $U$ onto $Z(U)$ and that $U$ is the domain of local coordinates $x_{j}(1 \leq j \leq m)$ all vanishing at a "central point" which will be denoted by $O$. We will suppose $Z(o)=o$ and denote by $Z_{x}$ the Jacobian matrix of the $Z^{j}$ with respect to the $x_{k}$. Substitution of $Z_{x}(o)^{-1} Z(x)$ for $Z(x)$ will allow us to assume that $Z_{x}(o)=$ the identity matrix. Since the differential of $\operatorname{Im} Z_{x}$ is zero at the origin, after shrinking $U$ if necessary, we can find a number $K, 0<K<1$, such that for all $x, y$ in $U$ and for all $\xi$ in $R_{m}$.

$$
\left|\operatorname{Im} Z_{x}^{*}(x) \xi\right| \leq K\left|\operatorname{Re} Z_{x}^{*}(x) \xi\right| \quad \text { and }
$$

$$
\begin{aligned}
& \operatorname{Re}\left(\sqrt{-1} Z_{x}^{*}(x) \xi \cdot(Z(x)-Z(y))-\left\langle Z_{x}^{*}(x) \xi\right\rangle(Z(x)-Z(y))^{2}\right) \\
& \quad \leq-K|\xi||Z(x)-Z(y)|^{2}
\end{aligned}
$$

where $\langle\zeta\rangle^{2}=\zeta_{1}^{2}+\cdots+\zeta_{m}^{2}$ for $\zeta \in C_{m},|\operatorname{Re} \zeta|<|\operatorname{Im} \zeta|$.

Let $u$ be a compactly supported distribution in $U$. We shall refer to

$$
F(u, z, \zeta)=\int_{U} \exp \left(\sqrt{-1} \zeta(z-Z(y))-\langle\zeta\rangle(z-Z(y))^{2}\right) u(y) d Z(y)
$$

as the Fourier-Bros-Iagolnitzer (in short, FBI) transform of $u$ (see [1]). Here $z \in C^{m}, \zeta \in C_{m}$ with $|\operatorname{Im} \zeta|<|\operatorname{Re} \zeta|$.

In [1] the authors established a connection between the concept of the FBI transform and the notion of hypo-analytic wave front set. 
Their theorems that showed the equivalence between exponential decay in the FBI transform of $u$ and microlocal hypo-analyticity may be consolidated into the following:

THEOREM 3.1. The following two properties are equivalent:

(i) $u$ is hypo-analytic at $\left(0, \xi^{0}\right) \in T^{*} U \backslash\{0\}$.

(ii) There is an open neighborhood $V$ of 0 in $C^{m}$, a conic open neighborhood $\mathscr{C}_{0}$ of $\xi^{0}$ in $C_{m}$ and constants $c, r>0$ such that $|F(u, z, \zeta)| \leq c e^{-r|\zeta|}$ for all $z$ in $V$ and for all $\zeta$ in $\mathscr{C}_{0}$.

We emphasize here that in [1] the assumption that $\operatorname{Im} Z_{x}(0)=0$ was exploited in proving the sufficiency part, that is, the implication (ii) $\Rightarrow$ (i) of the above theorem. We don't know whether (ii) implies (i) at a point $(x, \xi) \in T^{*} U \backslash\{0\}$ without the additional hypothesis that $\operatorname{Im} Z_{x}(x)=0$.

In fact Theorem 3.1 does not give us any information regarding the points $(x, \xi) \in T^{*} U \backslash\{0\}$ unless $\operatorname{Im} Z_{x}(x)=0$. Here we would like to show that such an assumption is not needed for the necessity part of Theorem 3.1. More precisely, we have:

THEOREM 3.2. Suppose the distribution u of compact support is hypoanalytic at $\left(x_{0}, \xi^{0}\right) \in T^{*} U \backslash\{0\}$. Then there is an open neighborhood $V$ of $Z\left(x_{0}\right)$ in $C^{m}$, a conic open neighborhood $\mathscr{C}_{0}$ of $Z_{x}^{*}\left(x_{0}\right) \xi^{0}$ in $C_{m}$ and constants $c, r>0$ such that $|F(u, z, \zeta)| \leq c e^{-r|\zeta|}$ for all $z$ in $V$ and for all $\zeta$ in $\mathscr{C}_{0}$.

Proof. According to the definition, it suffices to prove the result when $u$ is the boundary value of a holomorphic function $f$ of tempered growth defined in a set of the form

$$
\left\{Z(x)+\sqrt{-1} Z_{x}(x) v: x+\sqrt{-1} v \in(W+\sqrt{-1} \Gamma),|v|<\delta_{0}\right\}
$$

where $W \subset U$ is an open neighborhood of $x_{0}, \delta_{0}$ a positive number and $\Gamma$ an acute open cone in $R_{m} \backslash\{0\}$ such that for very $v \in \Gamma, \xi^{0} \cdot v<0$. Thus for $\varphi \in C_{c}^{\infty}(W)$,

$$
\langle u, \varphi\rangle=\lim _{t \rightarrow+0} \int f\left(Z(x)+t \sqrt{-1} Z_{x}(x) v\right) \varphi(x) d Z(x), \quad v \in \Gamma .
$$

After contracting $\Gamma$ if necessary, we may assume that there is a number $c_{0}>0$ such that $\xi^{0} \cdot v \leq-c_{0}|v|\left|\xi^{0}\right|$ whenever $v$ is in $\Gamma$. We shall need the following lemma. 
LemMa 3.1. Suppose $u \in E^{\prime}(U)$ vanishes in an open neighborhood of $x_{0} \in U$. Then there is an open neighborhood $V$ of $Z\left(x_{0}\right)$ in $C^{m}, a$ conic neighborhood $\mathscr{C}$ of $\left\{Z_{x}^{*}\left(x_{0}\right) \xi: \xi \in R_{m} \backslash\{0\}\right\}$ in $C_{m}$ and constants $c, r$ such that $|F(u, z, \zeta)| \leq c e^{-r|\zeta|}$ for all $z$ in $V$ and for all $\zeta$ in $\mathscr{C}$.

Proof of Lemma 3.1. For $z \in C^{m}$ and $\zeta \in C_{m},|\operatorname{Im} \zeta|<|\operatorname{Re} \zeta|$ we consider the FBI

$$
F(u, z, \zeta)=\int \exp \left(\sqrt{-1} \zeta \cdot(z-Z(y))-\langle\zeta\rangle(z-Z(y))^{2}\right) u(y) d Z(y) .
$$

Let

$$
Q(z, \zeta, y)=\operatorname{Re}\left\{\sqrt{-1} \frac{\zeta}{|\zeta|} \cdot(z-Z(y))-\frac{\langle\zeta\rangle}{|\zeta|}(z-Z(y))^{2}\right\} .
$$

We first freeze $z$ to $Z\left(x_{0}\right)$ and $\zeta$ to $Z_{x}^{*}\left(x_{0}\right) \cdot \xi^{0}$ for some $\xi^{0} \in R_{m}$, $\left|\xi^{0}\right|=1$.

$$
\begin{aligned}
& Q\left(Z\left(x_{0}\right), Z_{x}^{*}\left(x_{0}\right) \xi^{0}, y\right)=\operatorname{Re}\left\{\sqrt{-1} \frac{Z_{x}^{*}\left(x_{0}\right) \xi^{0}}{\left|Z_{x}^{*}\left(x_{0}\right) \xi^{0}\right|}\left(Z\left(x_{0}\right)-Z(y)\right)\right. \\
& \left.-\frac{\left\langle Z_{x}^{*}\left(x_{0}\right) \xi^{0}\right\rangle}{\left|Z_{x}^{*}\left(x_{0}\right) \xi^{0}\right|}\left(Z\left(x_{0}\right)-Z(y)\right)^{2}\right\} \text {. }
\end{aligned}
$$

Condition (3.2) tells us that

$$
Q\left(Z\left(x_{0}\right), Z_{x}^{*}\left(x_{0}\right) \xi^{0}, y\right) \leq-K\left|Z\left(x_{0}\right)-Z(y)\right|^{2} .
$$

Suppose $d$ is a positive number such that $\left|y-x_{0}\right| \geq d$ whenever $y \in$ $\operatorname{Supp} u$.

Then in the support of $u, Q\left(Z\left(x_{0}\right), Z_{x}^{*}\left(x_{0}\right) \xi^{0}, y\right) \leq-K d^{2}$. By continuity, there are open neighborhoods $\tilde{V}$ of $Z\left(x_{0}\right)$ in $C^{m}$ and $\tilde{\mathscr{E}}$ of $Z_{x}^{*}\left(x_{0}\right) \xi^{0}$ in $C_{m}$ such that

$$
Q(z, \zeta, y) \leq-\frac{K d^{2}}{2} \text { for all } z \text { in } \tilde{V}, \zeta
$$

in $\tilde{\mathscr{C}}$. By compactness of the unit sphere in $R_{m}$, we may assume that the open set $\tilde{\mathscr{C}}$ contains the set $\left\{Z_{x}^{*}\left(x_{0}\right) \xi: \xi \in R_{m},|\xi|=1\right\}$.

Moreover, the homogeneity of $Q$ implies that there is a conic neighborhood $\mathscr{C}$ of $\left\{Z_{x}^{*}\left(x_{0}\right) \xi: \xi \in R_{m} \backslash\{0\}\right\}$ in $C_{m}$ such that

$$
\operatorname{Re}\left\{\sqrt{-1} \zeta \cdot(z-Z(y))-\langle\zeta\rangle(z-Z(y))^{2}\right\} \leq-\frac{K d^{2}}{2}|\zeta|
$$

whenever $z$ is in $\tilde{V}$ and $\zeta$ is in $\mathscr{C}$. This gives us the required decay of $F(u, z, \zeta)$. 
End of proof of Theorem 3.2. Let $g \in C_{c}^{\infty}(W), g \equiv 1$ near $x_{0}$. Since $(1-g) u$ vanishes near $x_{0}$, by Lemma 3.1 we know that $F((1-g) u, z, \zeta)$ decays exponentially in the sets of interest. Therefore, it suffices to show a similar decay for $F(g u, z, \zeta)$. Let $\chi \in$ $C_{c}^{\infty}(W), \chi \equiv 1$ near $x_{0}$ and supp $\chi \subset\{x: g(x) \equiv 1\}$. Fix $v \in \Gamma,|v|=$ 1 . When $s$ is a suitably small positive number, we can deform the contour of integration in $F(g u, z, \zeta)$ under the mapping:

$$
Z(y) \rightarrow \tilde{Z}(y)=Z(y)+\sqrt{-1} s Z_{y}(y) \chi(y) v .
$$

Thus

$$
\begin{aligned}
& F(g u, z, \zeta) \\
& \quad=\int_{U} \exp \left(\sqrt{-1} \zeta \cdot(z-\tilde{Z}(y))-\langle\zeta\rangle(z-\tilde{Z}(y))^{2}\right) f(\tilde{Z}(y)) \\
& \cdot g(y) d \tilde{Z}(y) .
\end{aligned}
$$

We focus on the quantity

$$
Q(z, \zeta, y, s)=\operatorname{Re}\left\{\sqrt{-1} \frac{\zeta}{|\zeta|} \cdot(z-\tilde{Z}(y))-\frac{\langle\zeta\rangle}{|\zeta|}(z-\tilde{Z}(y))^{2}\right\}
$$

and write it as $Q=Q_{1}+Q_{2}$ where

$$
Q_{1}(z, \zeta, y)=\operatorname{Re}\left\{\sqrt{-1} \frac{\zeta}{|\zeta|} \cdot(z-Z(y))-\frac{\langle\zeta\rangle}{|\zeta|} \cdot(z-Z(y))^{2}\right\}
$$

and

$$
\begin{aligned}
& Q_{2}(z, \zeta, y, s) \\
& =\operatorname{Re}\left\{\frac{\zeta}{|\zeta|} \cdot\left(s Z_{y}(y) \chi(y) v\right)\right. \\
& \left.\quad+\frac{\langle\zeta\rangle}{|\zeta|}\left[2 \sqrt{-1} s(z-Z(y)) \cdot\left(\chi(y) Z_{y}(y) v\right)+s^{2}\left|\chi(y) Z_{y}(y) v\right|^{2}\right]\right\} .
\end{aligned}
$$

We first consider these quantities when $z=Z\left(x_{0}\right), \zeta=Z_{x}^{*}\left(x_{0}\right) \cdot \xi^{0}$ and $y$ varies in the support of $g$. From (3.2) we have:

$$
Q_{1}\left(Z\left(x_{0}\right), Z_{x}^{*}\left(x_{0}\right) \xi^{0}, y, s\right) \leq-\kappa\left|Z\left(x_{0}\right)-Z(y)\right|^{2} .
$$

To estimate $Q_{2}\left(Z\left(x_{0}\right), Z_{x}^{*}\left(x_{0}\right) \xi^{0}, y, s\right)$, we note that for $s$ sufficiently small, say $0<s \leq s_{0}$ :

$$
\begin{aligned}
& Q_{2}\left(Z\left(x_{0}\right), Z^{*}\left(x_{0}\right) \xi^{0}, x_{0}, s\right) \\
& \quad=\operatorname{Re}\left\{\frac{s\left(\xi^{0} \cdot v\right)}{\left|Z_{x}^{*}\left(x_{0}\right) \xi^{0}\right|}+\frac{\left\langle Z_{x}^{*}\left(x_{0}\right) \xi^{0}\right\rangle}{\left|Z_{x}^{*}\left(x_{0}\right) \xi^{0}\right|} s^{2}\left|Z_{x}\left(x_{0}\right) v\right|^{2}\right\} \leq-s c_{0} / 4 .
\end{aligned}
$$


Therefore, by continuity we can find a number $d>0$ satisfying:

$$
\left|y-x_{0}\right| \leq d \Rightarrow Q_{2}\left(Z\left(x_{0}\right), Z_{x}^{*}\left(x_{0}\right) \xi^{0}, y, s\right) \leq-s c_{0} / 4 .
$$

We may assume that $\chi(y)=1$ whenever $\left|y-x_{0}\right| \leq d$. On the other hand, whatever $y$,

$$
Q_{2}\left(Z\left(x_{0}\right), Z_{x}^{*}\left(x_{0}\right) \xi^{0}, y, s\right) \leq 4 s \chi(y)\left(\left|Z\left(x_{0}\right)-Z(y)\right|+s\right) .
$$

Hence when $\left|y-x_{0}\right| \leq d$,

$$
Q\left(Z\left(x_{0}\right), Z_{x}^{*}\left(x_{0}\right) \xi^{0}, y, s\right) \leq-\kappa\left|Z\left(x_{0}\right)-Z(y)\right|^{2}-s c_{0} / 2,
$$

while when $\left|y-x_{0}\right| \geq d$ then $\left|Z(y)-Z\left(x_{0}\right)\right| \geq d$ so that

$$
\begin{aligned}
& Q\left(Z\left(x_{0}\right), Z_{x}^{*}\left(x_{0}\right) \xi^{0}, y, s\right) \\
& \quad \leq-\kappa d\left|Z\left(x_{0}\right)-Z(y)\right|+4 s \chi(y)\left(\left|Z\left(x_{0}\right)-Z(y)\right|+s\right) .
\end{aligned}
$$

Therefore, by choosing $s$ small in comparison with $d$, we get a positive number $\delta$ such that

$$
Q\left(Z\left(x_{0}\right), Z_{x}^{*}\left(x_{0}\right) \xi^{0}, y, s\right) \leq-\delta, \quad y \in \operatorname{Supp} g .
$$

By continuity, there are open neighborhoods $\tilde{V}$ of $Z\left(x_{0}\right)$ in $C^{m}$ and $\tilde{\mathscr{C}}$ of $Z_{x}^{*}\left(x_{0}\right) \xi^{0}$ in $C_{m}$ such that $Q(z, \zeta, y, s) \leq-\delta / 2$ for all $z \in \tilde{V}, \zeta \in \tilde{\mathscr{C}}$ and for all $y \in \operatorname{Supp} g$. Now $Q$ is positive homogeneous of degree 0 in $\zeta$. Therefore, there is an open conic neighborhood $\mathscr{C}$ of $Z_{x}^{*}\left(x_{0}\right) \xi^{0}$ in $C_{m}$ such that

$$
\operatorname{Re}\left\{\sqrt{-1} \zeta \cdot(z-\tilde{Z}(y))-\langle\zeta\rangle(z-\tilde{Z}(y))^{2}\right\} \leq-\frac{\delta}{2}|\zeta|
$$

whenever $z$ is in $\tilde{V}$ and $\zeta$ in $\mathscr{C}$. From this, we get the required decay of $F(u, z, \zeta)$.

Proof of Theorem 2.1. For $x, y, \zeta \in C^{m}$, let

$$
\psi(x, y, \zeta)=(y-x) \frac{\zeta}{|\zeta|}+\frac{\sqrt{-1}}{2} \frac{\langle\zeta\rangle}{|\zeta|}(y-x)^{2} .
$$

The Cauchy-Kovalevska Theorem implies that there exists a holomorphic function $\phi=\phi(t, x, y, \zeta)$ such that

$$
\begin{gathered}
\frac{\partial \phi}{\partial t}(t, x, y, \zeta)=p\left(x,-\phi_{x}(t, x, y, \zeta)\right), \\
\phi(0, x, y, \zeta)=\psi(x, y, \zeta) .
\end{gathered}
$$

Since $\gamma(t)$ is a bicharacteristic for $P$, we have:

(i) $\phi\left(t, \tilde{x}(t), 0, \xi^{0}\right)=0$,

(ii) $\phi_{x}\left(t, \tilde{x}(t), 0, \xi^{0}\right)=-\tilde{\xi}(t)$, 
(iii) $\operatorname{Im} \phi_{x x}\left(t, \tilde{x}(t), 0, \xi^{0}\right)>0$.

Now (iii) clearly holds when $t=0$. Therefore, this will persist for $t$ small enough. To see (ii), we note that

$$
\frac{d}{d t}\left(\phi_{x}\left(t, \tilde{x}(t), 0, \xi^{0}\right)\right)=\phi_{x t}+\phi_{x x} \frac{d \tilde{x}}{d t}
$$

and (3.3) implies that $\phi_{t x}=p_{x}\left(x,-\phi_{x}\right)-p_{\zeta}\left(x,-\phi_{x}\right) \phi_{x x}$. It follows that

$$
\begin{aligned}
\frac{d}{d t}( & \left.-\phi_{x}\left(t, \tilde{x}(t), 0, \xi^{0}\right)\right) \\
= & -p_{x}\left(\tilde{x}(t),-\phi_{x}\left(t, \tilde{x}(t), 0, \xi^{0}\right)\right) \\
& +p_{\xi}\left(\tilde{x}(t),-\phi_{x}\left(t, \tilde{x}(t), 0, \xi^{0}\right)\right) \phi_{x x}\left(t, \tilde{x}(t), 0, \xi^{0}\right) \\
& -\phi_{x x}\left(t, \tilde{x}(t), 0, \xi^{0}\right) \frac{d \tilde{x}}{d t} .
\end{aligned}
$$

$\tilde{\xi}(t)$ also satisfies the same differential equation and $\phi_{x}\left(0,0,0, \xi^{0}\right)=$ $-\tilde{\xi}(0)$.

Hence $\phi_{x}\left(t, \tilde{x}(t), 0, \xi^{0}\right)=-\tilde{\xi}(t)$.

To see (i), we observe that

$$
\begin{aligned}
& \frac{d}{d t} \phi\left(t, \tilde{x}(t), 0, \xi^{0}\right) \\
& \quad=\phi_{t}+\phi_{x} \frac{d \tilde{x}}{d t}=\phi_{t}-\tilde{\xi}(t) \frac{\partial p}{\partial \zeta} \quad \text { and this in turn by }(3.3) \\
& \quad=p(\tilde{x}(t), \tilde{\xi}(t))-\tilde{\xi}(t) \frac{\partial p}{\partial \zeta}(\tilde{x}(t), \tilde{\xi}(t)) \\
& \quad=p(\tilde{x}(t), \tilde{\xi}(t))-k p(\tilde{x}(t), \tilde{\xi}(t))=0 .
\end{aligned}
$$

Also $\phi\left(0, \tilde{x}(0), 0, \xi^{0}\right)=0$. Hence $\phi\left(t, \tilde{x}(t), 0, \xi^{0}\right)=0$.

Since $P$ is of principal type, we can construct an analytic amplitude $a=\sum_{l=0}^{\infty} a_{l} \lambda^{-l}$ satisfying

$$
\left(D_{t}+\frac{{ }^{t} p\left(z, D_{z}\right)}{\lambda^{k-1}}\right) e^{i \lambda \phi(t, z, y, \zeta)} a(t, z, y, \zeta, \lambda)=0
$$

approximately and $a(0, z, y, \zeta, \lambda)=1$. The error in the approximation has an exponential decay. Indeed, substitution of $a=\sum_{l=0}^{\infty} a_{l} \lambda^{-l}$ in (3.4) gives the transport equations:

$$
\begin{array}{ll}
L a_{0}=0, & \left.a_{0}\right|_{t=0}=1, \\
L a_{1}+f_{1}\left(a_{0}\right)=0, & \left.a_{1}\right|_{t=0}=0, \\
\quad \vdots & \\
L a_{n}+f_{n}\left(a_{0}, \ldots, a_{n-1}\right)=0, & \left.a_{n}\right|_{t=0}=0,
\end{array}
$$


where

$$
L=\frac{\partial}{\partial t}+\sum_{j=1}^{m} \frac{\partial p^{t}}{\partial \zeta_{j}}\left(x, \phi_{x}\right) \frac{\partial}{\partial x_{j}}+s(x, y, \zeta)
$$

with $s$ holomorphic and $f_{n}\left(a_{0}, \ldots, a_{n-1}\right)$ a linear expression with holomorphic coefficients of the derivatives of $a_{0}, \ldots, a_{n-1}$. These equations can be solved successively in a complex domain independent of $n$. The fact that $a$ becomes an analytic amplitude is shown for example in [5].

Suppose now $\gamma\left(t_{0}\right)=\left(x\left(t_{0}\right), \xi\left(t_{0}\right)\right) \notin W f_{\text {ha }} u$ for some $t_{0}$. Then we claim that

$$
F\left(t_{0}, z, \zeta, \lambda\right)=\int e^{\sqrt{-1} \lambda \phi\left(t_{0}, Z(x), z, \zeta\right)} a\left(t_{0}, Z(x), z, \zeta, \lambda\right) u(x) d Z(x)
$$

decays exponentially (i.e. $\left|F\left(t_{0}, z, \zeta, \lambda\right)\right| \leq c_{1} e^{-c_{2} \lambda}, \lambda>0$ ) for $z$ near 0 and $\zeta$ near $\xi^{0}$.

To prove this assertion, we look at the Taylor's expansion of $\phi$ around the point $\left(t_{0}, \tilde{x}\left(t_{0}\right), 0, \xi^{0}\right)$.

For $x, z \in C^{m} \zeta \in C_{m}$ and $t_{0}$ fixed, we have:

$$
\begin{aligned}
\phi\left(t_{0}, x, z, \zeta\right)= & \phi\left(t_{0}, \tilde{x}\left(t_{0}\right), 0, \xi^{0}\right)+\phi_{x}\left(t_{0}, \tilde{x}\left(t_{0}\right), 0, \xi^{0}\right)\left(x-\tilde{x}\left(t_{0}\right)\right) \\
& +\phi_{z}\left(t_{0}, \tilde{x}\left(t_{0}\right), 0, \xi^{0}\right) z+\phi_{\zeta}\left(t_{0}, \tilde{x}\left(t_{0}\right), 0, \xi^{0}\right)\left(\zeta-\xi^{0}\right) \\
& +\phi_{x x}\left(t_{0}, \tilde{x}\left(t_{0}\right), 0, \xi^{0}\right)\left(x-\tilde{x}\left(t_{0}\right)\right)^{2}+\cdots .
\end{aligned}
$$

Since $\phi\left(t_{0}, \tilde{x}\left(t_{0}\right), 0, \xi^{0}\right)=0$ and $\phi_{x}\left(t_{0}, \tilde{x}\left(t_{0}\right), 0, \xi^{0}\right)=-\tilde{\xi}\left(t_{0}\right)$, we can write

$$
\begin{aligned}
\phi\left(t_{0}, x, z, \zeta\right)=\tilde{\xi}\left(t_{0}\right) \cdot\left(\tilde{x}\left(t_{0}\right)-x\right) \\
\\
+\phi_{x x}\left(t_{0}, \tilde{x}\left(t_{0}\right), 0, \xi^{0}\right)\left(\tilde{x}\left(t_{0}\right)-x\right)^{2}+R\left(t_{0}, x, z, \zeta\right)
\end{aligned}
$$

where $R\left(t_{0}, x, z, \zeta\right)$ is a sum of terms each of which has at least one of $\zeta-\xi^{0}, z$ or $\left(\tilde{x}\left(t_{0}\right)-x\right)^{3}$ as a factor. Thus we have:

$$
\begin{aligned}
\phi\left(t_{0}, Z(x), z, \zeta\right)= & Z_{x}^{*}\left(x\left(t_{0}\right)\right) \xi\left(t_{0}\right) \cdot\left(Z\left(x\left(t_{0}\right)\right)-Z(x)\right) \\
& +\phi_{x x}\left(t_{0}, Z\left(x\left(t_{0}\right)\right), 0, \xi^{0}\right)\left(Z\left(x\left(t_{0}\right)\right)-Z(x)\right)^{2} \\
& +R\left(t_{0}, Z(x), z, \zeta\right) .
\end{aligned}
$$

We recall that $\operatorname{Im} \phi_{x x}\left(t_{0}, Z\left(x\left(t_{0}\right)\right), 0, \xi^{0}\right)>0$. Since $\left(x\left(t_{0}\right), \xi\left(t_{0}\right)\right) \notin$ $W F_{\text {ha }} u$, Theorem 3.2 implies that

$$
\begin{array}{r}
\int \exp \left(\sqrt { - 1 } \lambda \left[Z_{x}^{*}\left(x\left(t_{0}\right)\right) \xi\left(t_{0}\right) \cdot\left(Z\left(x\left(t_{0}\right)\right)-Z(x)\right)\right.\right. \\
\left.+\phi_{x x}\left(t_{0}, Z\left(x\left(t_{0}\right)\right), 0, \xi^{0}\right)\left(Z\left(x\left(t_{0}\right)\right)-Z(x)\right)^{2}\right] \\
\cdot a\left(t_{0}, Z(x), z, \zeta, \lambda\right) u(x) d Z(x)
\end{array}
$$


decays exponentially. In fact, the decay persists if we replace $Z_{x}^{*}\left(x\left(t_{0}\right)\right) \xi\left(t_{0}\right)$ by $\zeta$ and $Z\left(x\left(t_{0}\right)\right)$ by $\omega$ as long as they are sufficiently close to $Z_{x}^{*}\left(x\left(t_{0}\right)\right) \xi\left(t_{0}\right)$ and $Z\left(x\left(t_{0}\right)\right)$ respectively. Moreover, $R\left(t_{0}, Z\left(x\left(t_{0}\right)\right), 0, \xi^{0}\right)=0$. It follows that there are open neighborhoods $U_{1}, U_{2}$ of 0 and $\xi^{0}$ respectively such that if $z \in U_{1}$ and $\zeta \in U_{2}$, then

$$
\int e^{\sqrt{-1} \lambda \phi\left(t_{0}, Z(x), z, \zeta\right)} a\left(t_{0}, Z(x), z, \zeta, \lambda\right) u(x) d Z(x)
$$

decays exponentially uniformly in $U_{1} \times U_{2}$.

In the above considerations, the open set $U$ may have to be contracted. However, the contraction is independent of the distribution $u$.

Now let

$$
F(t, z, \zeta, \lambda)=\int e^{\sqrt{-1} \lambda \phi(t, Z(x), z, \zeta)} a(t, Z(x), z, \zeta, \lambda) u(x) d Z(x) .
$$

We have:

$$
\begin{aligned}
\left|\frac{\partial}{\partial t} F(t, z, \zeta, \lambda)\right| & =\left|\int \frac{\partial}{\partial t}\left(e^{\sqrt{-1} \lambda \phi} a\right) u(x) d Z(x)\right| \\
& =\frac{1}{\lambda^{k-1}}\left|\int{ }^{t} P\left(e^{\sqrt{-1} \lambda \phi} a\right) u(x) d Z(x)\right| \\
& =\frac{1}{\lambda^{k-1}}\left|\int e^{\sqrt{-1} \lambda \phi} a P u(x) d Z(x)\right| .
\end{aligned}
$$

Since $P u$ is hypo-analytic at each point $(x(t), \xi(t))$, our arguments imply that for each $t$, the last integral decays exponentially for $z$ near 0 and $\zeta$ near $\xi^{0}$. Moreover, this decay persists in a compact set of $t$ 's. It follows that there exist positive constants $c_{1}, c_{2}$ and open neighborhoods $W_{1}, W_{2}$ of 0 and $\xi^{0}$ respectively such that

$$
\left|\frac{\partial}{\partial t} F(t, z, \zeta, \lambda)\right| \leq c_{1} e^{-c_{2} \lambda} \quad \text { for } \lambda>0,
$$

$z \in W_{1}, \zeta \in W_{2}$ and $0 \leq t \leq t_{0}$. Since we also had a similar decay for $F\left(t_{0}, z, \zeta, \lambda\right)$, it follows that $|F(0, z, \zeta, \lambda)| \leq d_{1} e^{-d_{2} \lambda}$ for some positive constants $d_{1}, d_{2}$ and $(z, \zeta)$ near $\left(0, \xi^{0}\right)$. But

$$
\begin{aligned}
F(0, z, \zeta, \lambda)=\int \exp (\sqrt{1} \lambda[ & (z-Z(x)) \frac{\zeta}{|\zeta|} \\
& \left.\left.+\sqrt{-1} \frac{\langle\zeta\rangle}{|\zeta|}(z-Z(x))^{2}\right]\right) u(x) d Z(x) .
\end{aligned}
$$

Therefore, by Theorem 3.1 of the previous section $\left(0, \xi^{0}\right) \notin W F_{\mathrm{ha}} u$. We have thus shown that there is an open neighborhood $N$ in $\gamma$ of 
$\left(0, \xi^{0}\right), N$ independent of the distribution $u$ such that $(x, \xi) \in N$ and $(x, \xi) \notin W F_{\mathrm{ha}} u \Rightarrow\left(0, \xi^{0}\right) \notin W F_{\mathrm{ha}} u$. This means that $W F_{\mathrm{ha}} u \cap \gamma$ is open in $\gamma$. But this set is always closed. Since $\gamma$ is connected, the theorem follows.

Acknowledgments. It is a pleasure to express my gratitude to Professor Francois Treves for his advice and encouragement during his direction of my dissertation [2] which is the source of this work. The author also thanks the referee for his helpful suggestions.

\section{REFERENCES}

[1] M. S. Baouendi, C. H. Chang and F. Treves, Microlocal hypo-analyticity and extensions of CR functions, J. Differential Geom., 18 (1983), 331-391.

[2] S. Berhanu, Hypo-analytic pseudodifferential operators, Dissertation, Rutgers University (1987).

[3] N. Hanges, Propagation of analyticity along real bicharacteristics, Duke Math. J., 49 (1981), 269-277.

[4] L. Hormander, Linear differential operators, Proc. Internat. Congress Math. (Nice, 1970), Vol. 1, Gauthier-Villars, Paris, 1971, pp. 121-133.

[5] J. Sjostrand, Propagation of analytic singularities for second order Dirichlet problems III, Comm. P. D. E., 6 (5) (1981), 499-567.

Received November 30, 1987 and in revised form May 17, 1988.

TEMPle UNIVERSITY

Philadelphia, PA 19122 